\title{
Overexpression of IncRNA NEAT1 mitigates multidrug resistance by inhibiting ABCG2 in leukemia
}

\author{
CAIHUA GAO $^{1 *}$, JIANYING ZHANG ${ }^{2 *}$, QINGYAN WANG $^{1}$ and CHUNHUA REN ${ }^{3}$ \\ Departments of ${ }^{1}$ Medical Services and ${ }^{2}$ Emergency; ${ }^{3}$ Happy Sisters Family Service Centre, \\ Weihai Maternal and Child Health Hospital, Weihai, Shandong 264200, P.R. China
}

Received June 17, 2015; Accepted April 29, 2016

DOI: $10.3892 / \mathrm{ol} .2016 .4738$

\begin{abstract}
Leukemia is a heterogeneous clonal disorder in which early hematopoietic cells fail to differentiate and do not undergo programmed cell death or apoptosis. Less than one-third of adult patients with leukemia are managed using current therapies due to the emergence of multidrug resistance (MDR), emphasizing the need for newer and more robust approaches. Recent reports have suggested that long non-coding RNAs (lncRNAs) contribute to selective gene expression and, hence, could be manipulated effectively to halt the progression of cancer. However, little is known regarding the role of lncRNA in leukemia. Nuclear paraspeckle assembly transcript 1 (NEAT1) is a nuclear-restricted lncRNA involved in the pathogenesis of certain types of cancer. Deregulated expression of NEAT1 has been reported in a number of human malignancies, including leukemia and other solid tumors. The present study aimed to characterize the role of NEAT1 in the regulation of MDR in leukemia. Using reverse transcription-quantitative polymerase chain reaction, it was demonstrated that NEAT1 messenger RNA (mRNA) expression levels were significantly downregulated in leukemia patient samples compared with those from healthy donors. Furthermore, NEAT1 mRNA expression was repressed in a number of leukemia cell lines, including K562, THP-1, HL-60 and Jurkat cells, compared with peripheral white blood control cells, consistent with the expression observed in patients with leukemia. In addition, the transfection of a NEAT1 overexpression plasmid into K562 and THP-1 leukemia cell lines alleviated MDR induced by cytotoxic agents, such as Alisertib and Bortezomib, through inhibition of ATP-binding cassette G2. Although more robust studies are warranted, the
\end{abstract}

Correspondence to: Dr Caihua Gao, Department of Medical Services, Weihai Maternal and Child Health Hospital, 51 Guangming Road, Weihai, Shandong 264200, P.R. China

E-mail: gch.21999@163.com

*Contributed equally

Key words: leukemia, long non-coding RNA, nuclear paraspeckle assembly transcript 1 , multidrug resistance current findings provide the basis for the use of NEAT1 as a novel promising target in the treatment of leukemia.

\section{Introduction}

Leukemia is a malignant clonal disorder of the hematopoietic system with high heterogeneity and poor prognosis. In recent years, although the incidence of leukemia has increased gradually (1), the exact oncogenic mechanisms underlying leukemia have not been fully characterized. In leukemia, cancer cells with multidrug resistance (MDR) to chemotherapeutic drugs significantly reduce the efficacy of cancer chemotherapy (2). Various mechanisms are involved in the MDR of cancer, including the induction of the anti-apoptotic machinery, an increase in intracellular drug efflux and a reduction in drug uptake (3). Overexpression of ATP-binding cassette (ABC) transporters, particularly $\mathrm{ABCB} 1, \mathrm{ABCC} 1$ and $\mathrm{ABCG} 2$, are one of most common reasons for the development of MDR in cancer cells (4-7).

Long non-coding RNAs (lncRNAs) are defined as transcripts containing $>200$ nucleotides that are typically transcribed by RNA polymerase II (8). Although the existence of IncRNAs has been known for several decades, the multiple functions of the IncRNA components of the noncoding genome have only been determined in the last 10 years. IncRNAs have important roles in maintaining cellular homeostasis during cell or tissue development, and are also critical factors in the pathophysiology of cancer (8-13). The molecular mechanisms of action of lncRNAs are highly variable; they function as molecular scaffolds for stabilizing protein-protein and protein-DNA interactions, and also act as decoys and guides that facilitate proximal and distal macromolecular interactions that typically occur on a genome template $(9,10)$.

Nuclear paraspeckle assembly transcript 1 (NEAT1), a structural scaffold for the formation of paraspeckles, has been identified as a nuclear-restricted lncRNA and is known to exist as two isoforms: $3.7 \mathrm{~kb}$ NEAT1_1 (MENع) and $23 \mathrm{~kb}$ NEAT1_2 $($ MEN $\beta)(14,15)$. NEAT1_1 is generally polyadenylated, while NEAT1_2 lacks a polyA tail (14). This lncRNA is an architectural component of a subnuclear structure termed the paraspeckle, which is suggested to be involved in the regulation of gene expression by retaining messenger RNAs (mRNAs) for nuclear editing $(15,16)$. However, the expression and function of NEAT1 in leukemia is still unclear. 
The aim of the present study was to characterize the involvement of NEAT1 in MDR associated with leukemia. NEAT1 was highly deregulated in samples from patients with leukemia compared with those from healthy donors, indicating a tumor suppressor function of NEAT1 in leukemia. Further evidence indicated that NEAT1 expression was repressed in leukemia cell lines, K562, THP-1, HL-60 and Jurkat. The transfection of a NEAT1 overexpression plasmid into K562 and THP-1 leukemia cells reduced MDR conferred by cytotoxic drugs, such as Alisertib and Bortezomib, which further enhanced the sensitivity of these cell lines to cytotoxic cell death.

\section{Materials and methods}

Evolutionary analysis. A previous study by Zeng et al reported that NEAT1 lncRNA is comprised of two isoforms, NEAT1_1 and NEAT1_2 (17). Therefore, the present study compared conservative sequences of the two isoforms using rVista 2.0 (http://rvista.dcode.org/) by performing evolutionary analysis of transcription factor binding sites.

Patient samples. A total of 36 patients (17 males and 19 females; median age, 53.1 years) with leukemia and 15 healthy donors (5 males and 11 females; median age, 26.0 years) from Weihai Maternal and Child Health Hospital (Weihai, China) were enrolled in the present study during February 2012 to December 2014. Written informed consent was provided by all patients, and the study protocol was approved by the Institutional Research Ethics Board of Weihai Maternal and Child Health Hospital. Inclusion criteria for patients were age 20-70 years, primary leukemia without other diseases and familial inherited diseases. The diagnosis of leukemia was determined by a combination of clinical, morphological, laboratory and immunophenotypic criteria, as defined by the World Health Organization classification (18). Peripheral white blood cell (PWBC) samples from $5 \mathrm{ml}$ whole blood of the patients or healthy donors were isolated with the Ficoll-Paque method, and then used for RNA extraction and subsequent analysis of NEAT1_1 and NEAT1_2, or stored at $-80^{\circ} \mathrm{C}$.

Cell culture. K562 chronic myelogenous leukemia cells, THP-1 acute monocytic leukemia cells, HL-60 primary myeloid leukemia cells and Jurkat $\mathrm{T}$ lymphocytic leukemia cells were purchased from Jinan CN-Cell Biotechnology, Co, Ltd. (Jinan, China). All cell lines were cultured in RPMI-1640 complete medium (Gibco; Thermo Fisher Scientific, Inc., Waltham, MA, USA) containing 10\% fetal calf serum (Gibco; Thermo Fisher Scientific, Inc.) at $37^{\circ} \mathrm{C}, 5 \% \mathrm{CO}_{2}$ and saturated humidity. Human PWBCs isolated from the healthy donors were cultured in RMPI-1640 complete medium and served as the negative control.

Reverse transcription-quantitative polymerase chain reaction $(R T-q P C R)$. Total RNA was isolated from the blood samples and cell lines using a TRIzol total RNA extraction kit purchased from Tiangen Biotech Co., Ltd. (Beijing, China), according to the manufacturer's protocol. RNA was eluted with $100 \mu 1$ RNase-free water and stored at $-80^{\circ} \mathrm{C}$. RT-qPCR was conducted using an iTaq Universal SYBR Green One-Step kit
Table I. Mean $\mathrm{IC}_{50}$ values in $\mathrm{K} 562$ and THP-1 cells.

\begin{tabular}{llll}
\hline & & \multicolumn{2}{c}{$\mathrm{IC}_{50}, \mu \mathrm{g} / \mathrm{ml}$} \\
\cline { 3 - 4 } Cell line & Group & \multicolumn{1}{c}{ Alisertib } & Bortezomib \\
\hline K562 & Empty & $0.21 \pm 0.03$ & $0.45 \pm 0.06$ \\
& NEAT1 & $0.09 \pm 0.02^{\mathrm{b}}$ & $0.28 \pm 0.04^{\mathrm{a}}$ \\
THP-1 & Empty & $0.35 \pm 0.05$ & $0.63 \pm 0.08$ \\
& NEAT1 & $0.22 \pm 0.04^{\mathrm{a}}$ & $0.33 \pm 0.07^{\mathrm{a}}$ \\
\hline
\end{tabular}

${ }^{\mathrm{a}} \mathrm{P}<0.05,{ }^{\mathrm{b}} \mathrm{P}<0.01$ vs. empty vector group. $\mathrm{IC}_{50}$, half maximal inhibitory concentration; NEAT1, nuclear paraspeckle assembly transcript 1.

(BioRad Laboratories, Inc., Hercules, CA, USA) in a StepOnePlus $^{\text {TM }}$ Real-Time PCR system (Applied Biosystems; Thermo Fisher Scientific, Inc.). The cycling conditions for the synthesis of complementary DNA were $25^{\circ} \mathrm{C}$ for $15 \mathrm{~min}, 42^{\circ} \mathrm{C}$ for $60 \mathrm{~min}$ and $80^{\circ} \mathrm{C}$ for $5 \mathrm{~min}$, while the qPCR cycling conditions were $50^{\circ} \mathrm{C}(2 \mathrm{~min}), 95^{\circ} \mathrm{C}(10 \mathrm{~min})$, and 40 cycles of $95^{\circ} \mathrm{C}(15 \mathrm{sec})$ and $60^{\circ} \mathrm{C}(1 \mathrm{~min})$. The primers used were as follows: Forward: 5'-AATTCTGTTACGTCATGT-3' and reverse, 5'-TTTCTA ATGAGTTTAGAACTCAAAC-3' for NEAT1_1; forward, 5'-CCTATCCGTTGGTTTGTG-3' and reverse, 5'-GAGGGT TGGGAACTTGTC-3' for NEAT1_2; and forward, 5'-CTG GGCTACACTGAGCACC-3' and reverse, 5'-AAGTGG TCGTTGAGGGCAATG-3' for glyceraldehyde 3-phosphate dehydrogenase (GAPDH). Housekeeping GAPDH mRNA was used as the control for comparing relative expression of RNAs. mRNA expression was quantified using the quantification cycle method (19).

Western blotting. Cells were washed twice in phosphate-buffered saline and lysed on ice for $30 \mathrm{~min}$ in radioimmunoprecipitation assay buffer (Cell Signaling Technology, Inc., Danvers, MA, USA). Protein extracts were separated by $8 \%$ sodium dodecyl sulfate-polyacrylamide gel electrophoresis. Proteins were then transferred to a polyvinylidenedifluoride membrane, and probed with mouse anti-human ABCG2 monoclonal antibody (dilution, 1:500; cat no. sc-58222; Santa Cruz Biotechnology, Inc., Dallas, TX, USA) and a goat anti-mouse polyclonal antibody against $\beta$-actin (dilution, 1:2,000; cat no. sc-1616; Santa Cruz Biotechnology). Western blot signals were visualized using a SuperSignal West Pico Chemiluminescent Substrate kit (Pierce; Thermo Fisher Scientific, Inc.).

Transfection. A NEAT1 overexpression plasmid, pcDNA3.1-NEAT1, was commercially constructed by Genechem Co., Ltd. (Shanghai, China), and empty pcDNA3.1 vector (which was a gift from Dr Tao Li, Department of Biology, Zhejiang Normal University, Jinhua, China) was used as the control. To establish cell lines with transient overexpression of NEAT1, K562 and THP-1 cells $\left(1 \times 10^{7} / \mathrm{ml}\right)$ were transfected with $10 \mu \mathrm{g}$ pcDNA3.1-NEAT1 plasmid or control pcDNA3.1 vector in a $0.2 \mathrm{~cm}$ cuvette (BTX Instrument Division; Harvard Apparatus, Inc., Holliston, MA, USA) using a ECM 830 Electroporator (BTX Instrument Division; Harvard Apparatus, Inc.) at $130 \mathrm{~V}$ for $20 \mathrm{msec}$. 
A

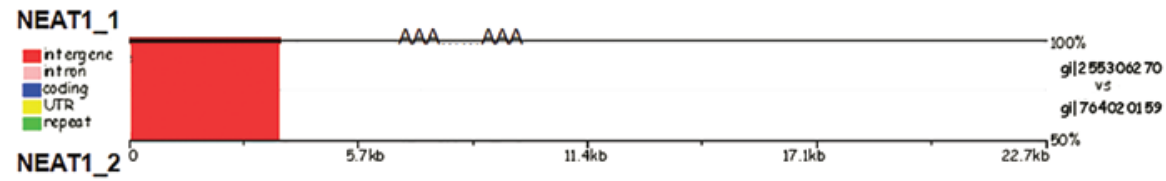

B
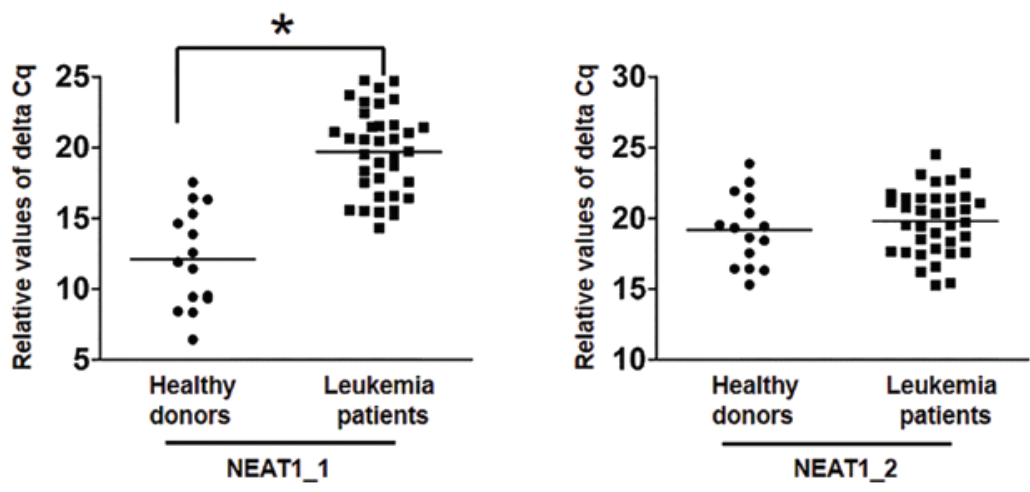

Figure 1. NEAT1 mRNA is downregulated in patients with leukemia. (A) Conserved sequences of NEAT1_1 and NEAT1_2 were analyzed using rVista 2.0. Schematic diagram indicating the conserved sequence between the two isoforms. Intergene, the nucleotides between two genes; intron, a segment of DNA that does not code for proteins and interrupts the sequence of genes; coding, a segment of DNA codes for proteins; repeat, the repetitive sequences; AAA, the adenylate residues at the 3 '-end of mRNA. (B) The mRNA expression levels of NEAT1_1 and NEAT1_2 were analyzed in sera from patients with leukemia by performing a reverse transcription-quantitative polymerase chain reaction. Sera from healthy donors were used as the control. Values were normalized to GAPDH. Data are presented mean \pm standard deviation from 36 patients with leukemia and 15 healthy donors. "P<0.05. NEAT1, nuclear paraspeckle assembly transcript 1; UTR, untranslated regions; Cq, quantification cycle.

Halfmaximal inhibitory concentration $\left(I C_{50}\right)$ detection. Apoptosis-inducing agents Alisertib (Celgene Corporation, Summit, NJ, USA) and Bortezomib (Shanghai Haoran Biotechnology, Co, Ltd., Shanghai, China) were utilized to determine the percentage of apoptotic cells using the 3-(4,5-dimethylthiazol2-yl)-2,5-diphenyltetrazolium bromide (MTT) assay. Briefly, K562 and THP-1 cell lines were collected in the logarithmic phase of growth and stained with trypan blue to determine cell viability using an inverted phase contrast microscope (CKX41; Olympus Corporation, Tokyo, Japan). Thereafter, the cell concentration was adjusted to $1 \times 10^{5}$ cells $/ \mathrm{ml}$. Each well of the 96-well culture plate was seeded with $100 \mu \mathrm{l}$ cells or with no cells (control groups). Following transfection with NEAT1 or control vectors, cells were cultured for $12 \mathrm{~h}$ with Alisertib $(0.1 \mu \mathrm{g} / \mathrm{ml})$ or Bortezomib $(0.1 \mu \mathrm{g} / \mathrm{ml})$. MTT reagent $(5 \mathrm{mg} / \mathrm{ml}, 10 \mu \mathrm{l} /$ well $)$ was added $4 \mathrm{~h}$ before termination of culture. Following incubation, cells were centrifuged at $153 \mathrm{x} \mathrm{g}$ for $5 \mathrm{~min}$ at $4^{\circ} \mathrm{C}$. The supernatant in each well was then removed, and the crystals were solubilized with $150 \mu \mathrm{l}$ dimethyl sulfoxide. Plates were shaken for $1 \mathrm{~min}$ and optical density (OD) was measured at $490 \mathrm{~nm}$ using a standard plate reader. The inhibition rate of cell proliferation was calculated as follows: Inhibition rate $(\%)=(1-\mathrm{OD}$ of the test / OD of the control) $\mathrm{x} 100 \%$. The $\mathrm{IC}_{50}$ was calculated by mid-efficacy analysis in K562 and THP-1 cells using the LOGIT method.

Flow cytometry assay. An Annexin V-fluorescein isothiocyanate (FITC)/propidium iodide (PI) double staining kit (BD Biosciences, San Jose, CA, USA) was used to detect apoptosis, according to the manufacturer's instructions. In brief, cells were transfected with different plasmids and treated with Alisertib or Bortezomib for $48 \mathrm{~h}$. Subsequently, the cells were collected via centrifugation at $153 \mathrm{x}$ g for $10 \mathrm{~min}$ at $4{ }^{\circ} \mathrm{C}$, stained with Annexin V-FITC/PI for $15 \mathrm{~min}$ at room temperature and subjected to flow cytometric analysis (BD LSRFortessa flow cytometer; BD Biosciences).

Statistical analysis. Statistical analysis was performed using SPSS version 11.0 for Windows (SPSS, Inc., Chicago, IL, USA). Mean differences between groups were compared with Student's t-test, while comparison of the different treatment groups to the control group was analyzed with analysis of variance. Data are presented as the mean \pm standard deviation of $\geq 3$ independent experiments, unless otherwise stated. $P<0.05$ was considered to indicate a statistically significant difference.

\section{Results}

Expression of NEAT1 mRNA is suppressed in patients with leukemia. The present study compared conservative sequences of the two isoforms by performing evolutionary analysis of transcription factor binding sites. The sequence alignment results demonstrated that there was a conserved sequence between the two isoforms (Fig. 1A). Furthermore, RT-qPCR was used to analyze the transcription level of NEAT1 isoforms in sera from patients with leukemia, and revealed that expression of the NEAT1_1 isoform was decreased compared with healthy donors while NEAT1_2 expression was not significantly different between the two cohorts $(\mathrm{P}<0.05$; Fig. 1B). The results suggest that NEAT1_1 may have a role as a tumor suppressor in the oncogenesis of leukemia.

NEAT1 mRNA is downregulated in leukemia cell lines. To further explore the detailed molecular functions of NEAT1, its expression was determined in four different leukemia cell lines, K562, THP-1, HL-60 and Jurkat, as well as human peripheral white blood cells (PWBC) isolated from healthy donors (negative control). The results showed that NEAT1 maintained 
A

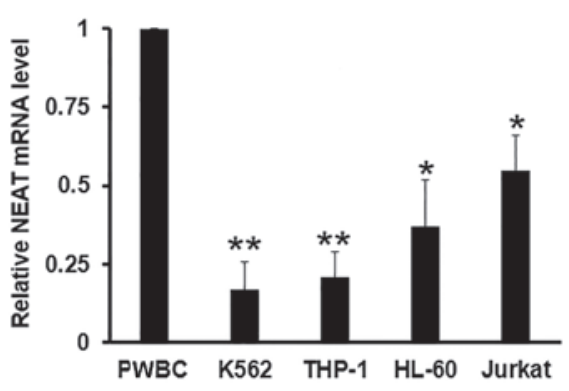

B

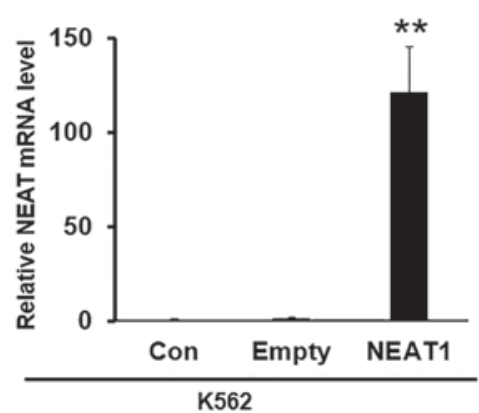

C

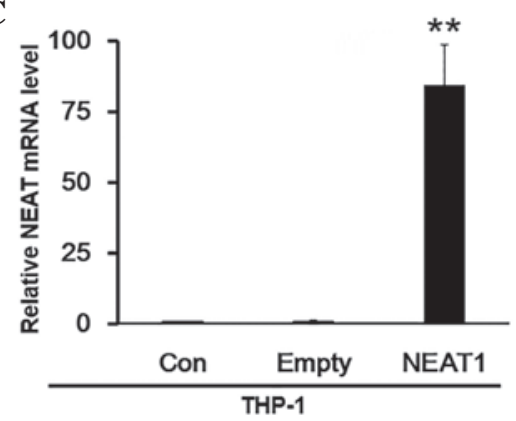

Figure 2. NEAT1 mRNA levels are decreased in a panel of leukemia cell lines. (A) The mRNA expression levels of NEAT1 were detected in K562, THP-1, HL-60 and Jurkat cells by reverse transcription-polymerase chain reaction (RT-qPCR). PWBC cells from healthy donors were used as the control. Values were normalized to GAPDH. (B and C) Representative experimental data showing the transfection efficiency of empty vector or NEAT1 overexpression plasmid in (B) K562 and (C) THP-1 cells, as verified by RT-qPCR. Data are presented as mean \pm standard deviation from three independent experiments. "P<0.05, ${ }^{* *} \mathrm{P}<0.01$ for K562, THP-1, HL-60 and Jurkat cell lines compared with PWBC control, or NEAT1 compared with empty vector. PWBC, peripheral white blood cells; Con, control; NEAT1, nuclear paraspeckle assembly transcript 1.
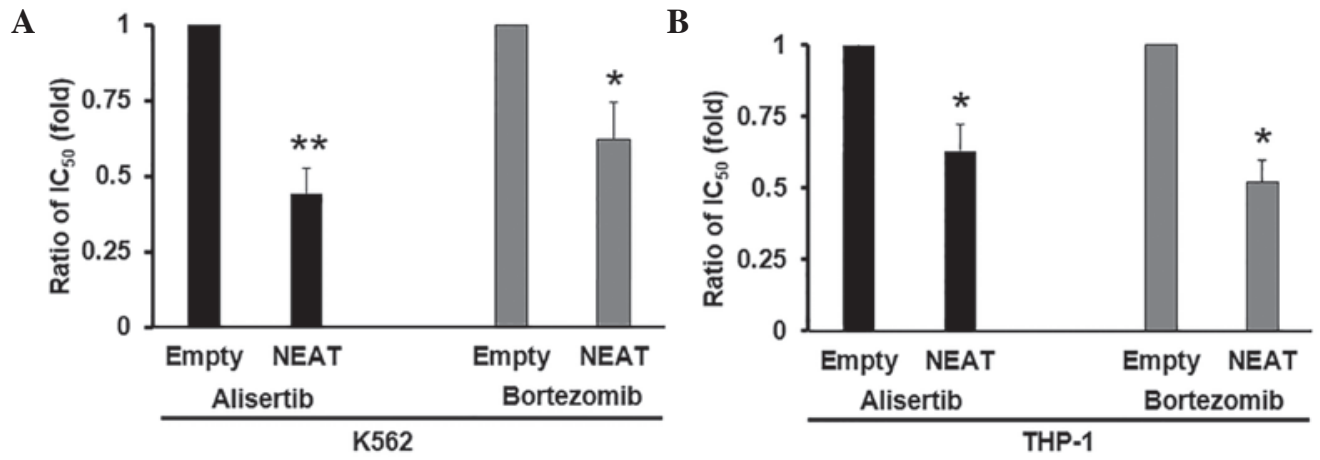

Figure 3. Overexpression of NEAT1 alleviates multidrug resistance in leukemia cell lines. The $\mathrm{IC}_{50}$ values of Alisertib and Bortezomib in (A) K562 and (B) THP-1 cells as determined by an MTT cell viability assay, following transfection with empty vector or NEAT1 overexpression plasmid. Data are presented as mean \pm standard deviation from at least three independent experiments. ${ }^{*} \mathrm{P}<0.05,{ }^{* * *} \mathrm{P}<0.01$ vs. empty vector. $\mathrm{IC}_{50}$, half maximal inhibitory concentration; NEAT1, nuclear paraspeckle assembly transcript 1.

A

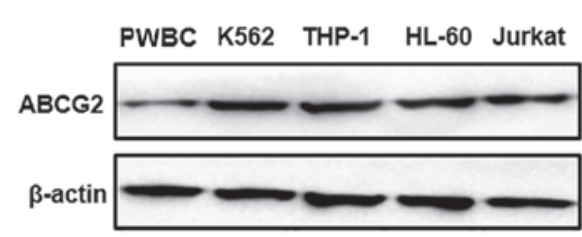

C
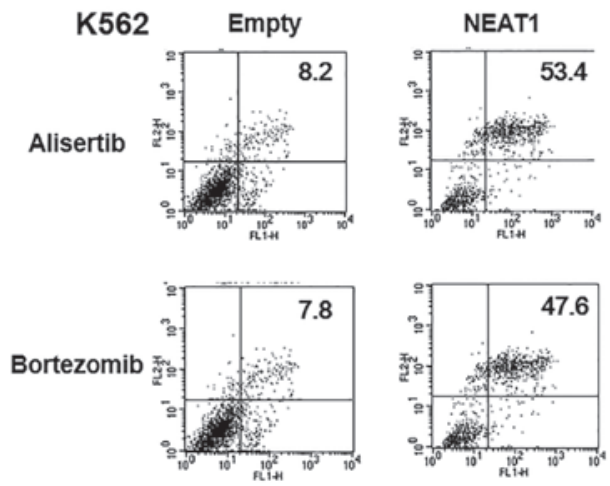

B

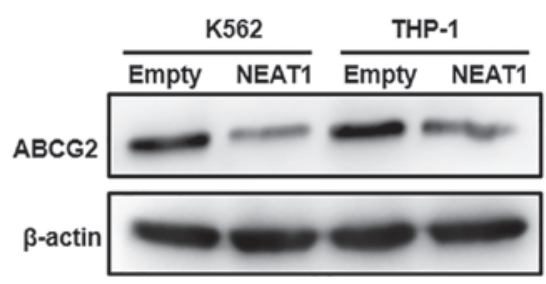

D
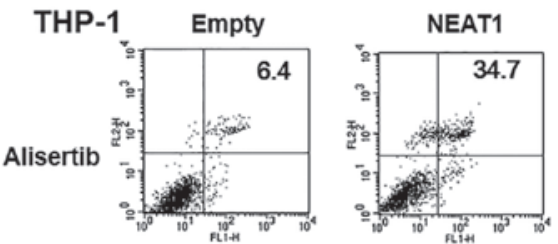
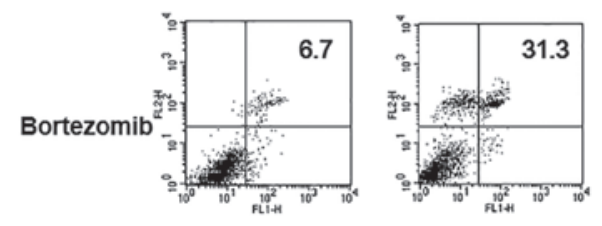

Figure 4. Overexpression of NEAT1 inhibits expression of ABCG2. (A) Basal expression levels of ABCG2 protein were detected by western blotting in four different leukemia cell lines and PWBC cells from healthy donors. (B) Protein expression of ABCG2 was also detected by western blotting in K562 and THP-1 cells transfected with NEAT1 overexpression plasmid. Cells transfected with empty vector were used as the control. (C and D) The apoptotic rate was detected using flow cytometry assays with an Annexin V-FITC and PI double staining kit in K562 and THP-1 cells. Both cells were transfected with empty vector or NEAT1 overexpression plasmid, and then treated with Alisertib or Bortezomib. PWBC, peripheral white blood cells; ABCG2, ATP-binding cassette G2; NEAT1, nuclear paraspeckle assembly transcript 1 . 
relatively high mRNA expression levels in PWBCs, but was significantly suppressed in leukemia cells compared with the control PWBCs (Fig. 2A).

Overexpression of NEAT1 overcomes MDR and enhances drug sensitivity. In order to validate whether the tumor suppressor function of NEAT1 is repressed during the transformation stages, overexpression of NEAT1 was induced by transfection with full-length NEAT1 plasmid. The efficiency of overexpression was validated by RT-qPCR. Notably, NEAT1 mRNA was upregulated at least 100-fold compared with cells transfected with empty vector (Fig. 2B and C). Next, in order to investigate the effects of NEAT1 on MDR in leukemia cells, cytotoxicity assays were performed with Alisertib and Bortezomib in K562 and THP-1 cells. The mean $\mathrm{IC}_{50}$ values are shown in Table I. Compared with K562 and THP-1 cells transfected with empty vector, cells overexpressed with NEAT1 exhibited significantly higher sensitivity to Alisertib and Bortezomib. NEAT1 overexpression decreased the $\mathrm{IC}_{50}$ values of Alisertib and Bortezomib in $\mathrm{K} 562$ ( $\mathrm{P}<0.01$ and $\mathrm{P}<0.05$, respectively; Fig. 3A) and THP-1 $(\mathrm{P}<0.05$; Fig. 3B) cells. Taken together, the current results suggest that NEAT1 overexpression significantly enhances the sensitivity of anti-leukemic drugs in culture.

NEAT1 overexpression inhibits ABCG2 transporter protein expression. It is well known that ABCG2 has important roles in MDR in leukemia $(20,21)$. Therefore, to evaluate the effects of NEAT1 on the MDR of leukemic cells, the expression level of ABCG2 was detected by western blotting. First, the ABCG2 expression level was verified in four different types of leukemia cell and normal PWBC cells. The expression of ABCG2 was markedly increased in leukemia cells compared with the control cells (Fig. 4A). Furthermore, when NEAT1 was overexpressed in K562 and THP-1 cells, the expression level of ABCG2 was significantly decreased compared with control cells transfected with empty vector, consistent with the changes in $\mathrm{IC}_{50}$ values observed in leukemia cells (Fig. 4B). Next, the role of NEAT1 in regulating MDR was determined by inducing apoptosis in K562 and THP-1 cells. The results demonstrated that low doses of Alisertib or Bortezomib did not induce apoptosis in either cell line transfected with empty vector. However, apoptosis induced by Alisertib (53.4 vs $8.2 \%$ for K562 cells and 47.6 vs $7.8 \%$ for THP-4 cells) and Bortezomib (34.7 vs $6.4 \%$ for K562 cells and 31.3 vs $6.7 \%$ for THP-4 cells) increased in both cell lines following overexpression of NEAT1 (Fig. 4C and D). Taken together, these data suggest that lncRNA NEAT1 may regulate apoptosis and MDR-associated ABCG2 protein expression in leukemia.

\section{Discussion}

The human transcriptome is composed of a large set of protein-coding mRNAs, as well as numerous non-coding transcripts that have structural, regulatory or unknown functions. Over the last decade, much attention has been focused on microRNAs (miRNAs), a class of small non-coding RNAs that are involved in various biological and pathological processes $(22,23)$. More recently, IncRNAs, generally defined as non-coding RNAs of $>200$ nt in length without known protein-coding function (24), have risen to prominence with central roles in a diverse range of functions $(25,26)$. A small number of studies suggest that lncRNAs derived from multiple tumors are overexpressed or underexpressed, influencing cancer growth, survival and migration or invasion (26-28). Furthermore, lncRNAs have been shown to be dysregulated in various types of cancer and several lncRNAs have been functionally associated with cancer cell differentiation $(14,29,30)$. Although an increasing number of lncRNAs have been characterized in epigenetic regulation and the prognosis of solid tumors, they have not been explored extensively in hematological malignancies, such as leukemia.

NEAT1 is a critical component of the paraspeckle structure, which is formed from a single, intergenic exon with two smaller conserved regions in mammalian lineage; however, it is not easily identified in other vertebrates (31). NEAT1 is proposed to be involved in the regulation of genetic expression through controlling the nuclear retention of mRNAs containing long inverted repeats. These mRNAs are capable of forming intramolecular double-stranded RNAs and causing translation repression as a result of adenosine-to-inosine editing $(16,32)$. In the current study, the expression and function of NEAT1 was explored in patients with leukemia, as well as in a panel of leukemia cell lines. NEAT1 mRNA expression was significantly decreased in sera from patients with leukemia and in leukemia cell lines compared with the high levels observed in normal healthy donor sera and PWBCs, respectively. Several studies have proposed that the expression of isoforms NEAT1_1 and NEAT1_2 are equally distributed in numerous tissues, such as the prostate, colon, pancreas and ovaries $(31,33)$. During the differentiation of embryonic stem cells and muscle cells, NEAT1 lncRNAs have been found to be upregulated $(14,16)$. A previous study reported that estrogen receptor $\alpha$-regulated NEAT1 lncRNA acted as a transcriptional regulator and promoted tumorigenesis leading to prostate adenocarcinoma in experimental animal models (34). Furthermore, NEAT1 expression was associated with oncogenic activity and metastatic progression in lung cancer $(35,36)$. However, downregulation of NEAT1 has been observed in the several other malignancies, including those affecting the liver, retina and esophagus (37). Furthermore, anomalous NEAT1 expression has been reported in various human malignancies, including acute promyelocytic leukemia, where NEAT1 repression was predominant in patients with leukemia caused by promyelocytic leukemia-retinoic acid receptor $\alpha(\operatorname{RAR} \alpha)$ gene translocation (17). In addition, time-lapse imaging experiments with NEAT1 constructs demonstrated the integral role of NEAT1 expression and paraspeckles formation at the NEAT1 locus (38). Despite a small number of studies revealing the composition and organization of the paraspeckle structure, its role in the pathogenesis of many malignancies are still unexplored.

Understanding the mechanisms involved in chemoresistance is crucial to developing therapies targeted at diseases that respond poorly to chemotherapy, such as like leukemia. Drugs, including tyrosine kinase inhibitors, are known to cause lysosomal degradation of ABCG2 and reverse the stemness properties of leukemic stem cells $(39,40)$. In a recent study, Liu et al reported that lncRNA maternally expressed gene 3 is significantly decreased in human lung adenocarcinoma and partially contributes to the cisplatin resistance of lung 
adenocarcinoma cells (41). Zhang et al showed that lncRNA plasmacytoma variant translocation-1 (PVT-1) was highly expressed in gastric cancer tissues from cisplatin-resistant patients and cisplatin-resistant gastric cancer cells; and overexpression of PVT1 in gastric carcinoma promoted the development of MDR (42). Furthermore, Hang et al demonstrated that high expression of IncRNA AK022798 induced by Notch1 resulted in the formation of cisplatin-resistant SGC7901 and $\mathrm{BGC} 823$ cells via regulating the expression of $\mathrm{ABCC} 1$ and $\mathrm{ABCB} 1$ (43). The current study utilized the overexpression of NEAT1 in K562 and THP-1 cell lines to determine the MDR function in response to two different anti-leukemic drugs. The results suggested that NEAT1 has a crucial role in decreasing MDR induced by Alisertib and Bortezomib, and sensitizing cells to anti-cancer drugs. Furthermore, western blot analysis supported that overexpression of NEAT1 could inhibit ABCG2 expression in leukemia cells. There is no evidence in the literature currently linking MDR and NEAT1 in leukemia. However, RAR $\alpha$ overexpression and associated MDR upregulation was reported to enhance differentiation of various leukemia cell lines (44). Taken together, these findings further highlight the importance of NEAT1 dysregulation in hematological malignancies, including leukemia.

In conclusion, the present study provides novel insights into the mechanisms regulating MDR in leukemia cell lines, and identified NEAT1 as a potential therapeutic target that could be manipulated to mitigate the occurrence of leukemia. The results of the current study indicate that, as NEAT1 is repressed in leukemic patients and cell lines, overexpression of NEAT1 may be a tangible strategy to inhibit ABCG2 and, thus, overcome chemoresistance associated with anti-leukemic drugs.

\section{References}

1. Coombs CC, Tavakkoli M and Tallman MS: Acute promyelocytic leukemia: Where did we start, where are we now and the future. Blood Cancer J 5: e304, 2015.

2. Ozben T: Mechanisms and strategies to overcome multiple drug resistance in cancer. FEBS Lett 580: 2903-2909, 2006.

3. Tsuruo T, Naito M, Tomida A, Fujita N, Mashima T, Sakamoto H and Haga N: Molecular targeting therapy of cancer: Drug resistance, apoptosis and survival signal. Cancer Sci 94: 15-21, 2003.

4. Fung KL and Gottesman MM: A synonymous polymorphism in a common MDR1 (ABCB1) haplotype shapes protein function. Biochim Biophys Acta 1794: 860-871, 2009.

5. Chen CJ, Chin JE, Ueda K, Clark DP, Pastan I, Gottesman MM and Roninson IB: Internal duplication and homology with bacterial transport proteins in the mdr1 (P-glycoprotein) gene from multidrug-resistant human cells. Cell 47: 381-389, 1986.

6. Tatsuta T, Naito M, Oh-hara T, Sugawara I and Tsuruo T: Functional involvement of P-glycoprotein in blood-brain barrier. J Biol Chem 267: 20383-20391, 1992.

7. Thiebaut F, Tsuruo T, Hamada H, Gottesman MM, Pastan I and Willingham MC: Cellular localization of the multidrug-resistance gene product P-glycoprotein in normal human tissues. Proc Natl Acad Sci USA 84: 7735-7738, 1987.

8. Flynn RA and Chang HY: Long noncoding RNAs in cell-fate programming and reprogramming. Cell Stem Cell 14: 752-761, 2014.

9. Rinn JL and Chang HY: Genome regulation by long noncoding RNAs. Annu Rev Biochem 81: 145-166, 2012.

10. Wang KC and Chang HY: Molecular mechanisms of long noncoding RNAs. Mol Cell 43: 904-914, 2011.

11. Huarte M and Rinn JL: Large non-coding RNAs: Missing links in cancer? Hum Mol Genet 19: R152-R161, 2010.

12. Lee JT: Epigenetic regulation by long noncoding RNAs. Science 338: 1435-1439, 2012.
13. Batista PJ and Chang HY: Long noncoding RNAs: Cellular address codes in development and disease. Cell 152: 1298-1307, 2013

14. Sunwoo H, Dinger ME, Wilusz JE, Amaral PP, Mattick JS and Spector DL: MEN epsilon/beta nuclear-retained non-coding RNAs are up-regulated upon muscle differentiation and are essential components of paraspeckles. Genome Res 19: 347-359, 2009.

15. Naganuma $\mathrm{T}$ and Hirose T: Paraspeckle formation during the biogenesis of long non-coding RNAs. RNA Biol 10: 456-461, 2013.

16. Chen LL and Carmichael GG: Altered nuclear retention of mRNAs containing inverted repeats in human embryonic stem cells: Functional role of a nuclear noncoding RNA. Mol Cell 35: 467-478, 2009.

17. Zeng C, Xu Y, Xu L, Yu X, Cheng J, Yang L, Chen S and Li Y: Inhibition of long non-coding RNA NEAT1 impairs myeloid differentiation in acute promyelocytic leukemia cells. BMC Cancer 14: 693, 2014.

18. Wadleigh $\mathrm{M}$ and Tefferi A: Classification and diagnosis of myeloproliferative neoplasms according to the 2008 World Health Organization criteria. Int J Hematol 91: 174-179, 2010.

19. Livak KJ and Schmittgen TD: Analysis of relative gene expression data using real-time quantitative PCR and the 2(-Delta Delta C(T)) Method. Methods 25: 402-408, 2001.

20. Rahgozar S, Moafi A, Abedi M, Entezar-E-Ghaem M, Moshtaghian J, Ghaedi K, Esmaeili A and Montazeri F: mRNA expression profile of multidrug-resistant genes in acute lymphoblastic leukemia of children, a prognostic value for $\mathrm{ABCA} 3$ and ABCA2. Cancer Biol Ther 15: 35-41, 2014.

21. Gromicho M, Dinis J, Magalhães M, Fernandes AR, Tavares P, Laires A, Rueff J and Rodrigues AS: Development of imatinib and dasatinib resistance: Dynamics of expression of drug transporters ABCB1, ABCC1, ABCG2, MVP and SLC22A1. Leuk Lymphoma 52: 1980-1990, 2011.

22. Bartel DP: MicroRNAs: Genomics, biogenesis, mechanism, and function. Cell 116: 281-297, 2004.

23. Ambros V: The functions of animal microRNAs. Nature 431: 350-355, 2004.

24. Saxena A and Carninci P: Long non-coding RNA modifies chromatin: Epigenetic silencing by long non-coding RNAs. Bioessays 33: 830-839, 2011.

25. Kaikkonen MU, Lam MT and Glass CK: Non-coding RNAs as regulators of gene expression and epigenetics. Cardiovasc Res 90: 430-440, 2011.

26. Tsai MC, Manor O, Wan Y, Mosammaparast N, Wang JK, Lan F, Shi Y, Segal E and Chang HY: Long noncoding RNA as modular scaffold of histone modification complexes. Science 329: 689-693, 2010.

27. Rinn JL, Kertesz M, Wang JK, Squazzo SL, Xu X, Brugmann SA, Goodnough LH, Helms JA, Farnham PJ, Segal E and Chang HY: Functional demarcation of active and silent chromatin domains in human HOX loci by noncoding RNAs. Cell 129: 1311-1323, 2007.

28. Gupta RA, Shah N, Wang KC, Kim J, Horlings HM, Wong DJ, Tsai MC, Hung T, Argani P, Rinn JL, et al: Long non-coding RNA HOTAIR reprograms chromatin state to promote cancer metastasis. Nature 464: 1071-1076, 2010.

29. Zhang H, Chen Z, Wang X, Huang Z, He Z and Chen Y: Long non-coding RNA: A new player in cancer. J Hematol Oncol 6: 37, 2013.

30. Zhuang Y, Wang X, Nguyen HT, Zhuo Y, Cui X, Fewell C, Flemington EK and Shan B: Induction of long intergenic non-coding RNA HOTAIR in lung cancer cells by type I collagen. J Hematol Oncol 6: 35, 2013.

31. Hutchinson JN, Ensminger AW, Clemson CM, Lynch CR, Lawrence JB and Chess A: A screen for nuclear transcripts identifies two linked noncoding RNAs associated with SC35 splicing domains. BMC Genomics 8: 39, 2007.

32. Naganuma T, Nakagawa S, Tanigawa A, Sasaki YF, Goshima N and Hirose T: Alternative 3'-end processing of long noncoding RNA initiates construction of nuclear paraspeckles. EMBO J 31: 4020-4034, 2012.

33. Sasaki YT, Ideue T, Sano M, Mituyama T and Hirose T: MENepsilon/beta noncoding RNAs are essential for structural integrity of nuclear paraspeckles. Proc Natl Acad Sci USA 106: 2525-2530, 2009.

34. Chakravarty D, Sboner A, Nair SS, Giannopoulou E, Li R, Hennig S, Mosquera JM, Pauwels J, Park K, Kossai M, et al: The oestrogen receptor alpha-regulated lncRNA NEAT1 is a critical modulator of prostate cancer. Nat Commun 5: 5383, 2014. 
35. Pan LJ, Zhong TF, Tang RX, Li P, Dang YW, Huang SN and Chen G: Upregulation and clinicopathological significance of long non-coding NEAT1 RNA in NSCLC tissues. Asian Pac J Cancer Prev 16: 2851-2855, 2015.

36. Zhao W, An Y, Liang Y and Xie XW: Role of HOTAIR long noncoding RNA in metastatic progression of lung cancer. Eur Rev Med Pharmacol Sci 18: 1930-1936, 2014.

37. Gibb EA, Vucic EA, Enfield KS, Stewart GL, Lonergan KM Kennett JY, Becker-Santos DD, MacAulay CE, Lam S, Brown CJ and Lam WL: Human cancer long non-coding RNA transcriptomes. PloS One 6: e25915, 2011.

38. Bergmann JH and Spector DL: Long non-coding RNAs: Modulators of nuclear structure and function. Curr Opin Cell Biol 26: 10-18, 2014

39. Wang F, Wang XK, Shi CJ, Zhang H, Hu YP, Chen YF and Fu LW: Nilotinib enhances the efficacy of conventional chemotherapeutic drugs in $\mathrm{CD} 34^{+} \mathrm{CD} 38^{-}$stem cells and $\mathrm{ABC}$ transporter overexpressing leukemia cells. Molecules 19 3356-3375, 2014.
40. Wang XK, He JH, Xu JH, Ye S, Wang F, Zhang H, Huang ZC, To KK and Fu LW: Afatinib enhances the efficacy of conventional chemotherapeutic agents by eradicating cancer stem-like cells. Cancer Res 74: 4431-4445, 2014.

41. Liu J, Wan L, Lu K, Sun M, Pan X, Zhang P, Lu B, Liu G and Wang Z: The long noncoding RNA MEG3 contributes to cisplatin resistance of human lung adenocarcinoma. PloS One 10: e0114586, 2015.

42. Zhang XW, Bu P, Liu L, Zhang XZ and Li J: Overexpression of long non-coding RNA PVT1 in gastric cancer cells promotes the development of multidrug resistance. Biochem Biophys Res Commun 462: 227-232, 2015.

43. Hang Q, Sun R, Jiang C and Li Y: Notch 1 promotes cisplatin-resistant gastric cancer formation by upregulating lncRNA AK022798 expression. Anticancer Drugs 26: 632-640, 2015.

44. Stromskaya TP, Rybalkina EY, Zabotina TN, Shishkin AA and Stavrovskaya AA: Influence of RARalpha gene on MDR1 expression and P-glycoprotein function in human leukemic cells. Cancer Cell Int 5: 15, 2005. 